\title{
Texture Based Pattern Classification
}

\author{
R.J.Bhiwani \\ Asst.Prof \\ B.N. College of Engineering, \\ Pusad. (M.S.)
}

\author{
M.A.Khan \\ Lect. Sel.Grd. \\ B.N. College of Engineering, \\ Pusad. (M.S.)
}

\author{
S.M.Agrawal \\ Lect. Sel.Grd. \\ B.N. College of Engineering, \\ Pusad. (M.S.)
}

\begin{abstract}
Texture can be observed in many natural and synthetic images from multispectral satellite images to the microscopic images of cell or tissue samples. Texture is an innate property of virtually all surfaces, the grain of wood, the weave of fabric, the pattern of crop in fields etc It contains important information about the structural arrangement of surfaces and their relationship to the surrounding environment. Since the textural properties of images appear to carry useful information, for discriminating purpose features have always been calculated for textures.

The paper deals with the pattern classification using texture features. The features used in the project are standard deviation and entropy of all parts obtained after $3^{\text {rd }}$ level decomposition using DWT. A mean feature vector is calculated and is used for classification. For classification distance similarity is used. The different distances used are Euclidean distance, Manhattan distance, Bray Curtis distance and Canberra distance. The efficiency of classification is calculated for each distance and is compared. The time required for each retrieval, in every distance method used, is also calculated.
\end{abstract}

\section{Keywords}

Texture, pattern classification, DWT, Euclidean distance, Bray Curtis distance, Manhattan distance \& Canberra distance.

\section{INTRODUCTION}

\subsection{Textures}

Texture is an important characteristic for analysis of many types of images. Texture is present in many, real as well as synthetic data, e.g. clouds, trees, bricks, hair, fabric etc. Despite its importance and ubiquity in image data, a formal approach or definition of texture analysis does not exist [1]. The texture discrimination approaches are adhoc. Texture is an innate property of virtually all surfaces the grain of wood, the weave of fabric, the pattern of crop in fields etc [9]. It contains important information about the structural arrangement of surfaces and their relationship to the surrounding environment.
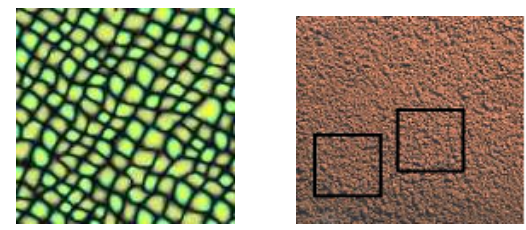

Figure 1.1 Texture image
Although it is quite easy for a human observer to recognize and describe in empirical terms, texture has been extremely adverse to precise definition and analysis by computer [2]. Since the textural properties of images appear to carry useful information, for discriminating purpose features have always been calculated for textures.

\subsection{Issues In Texture Analysis}

Major issues in texture analysis may be summarized as follows [3]:

1) Given a textured region, classify it in one of a finitely large number of texture classes.

2) To generate a description or model for a class of textured region.

3) Given an image having many distinct textured areas, determine the macro boundaries between different textured regions.

While, issue 1 involves the pattern recognition task of texture features estimation, issue 2 deals with identification of generate models of texture. The third important domain performs texture segmentation of an image. The paper deals with the issue 1 i.e. pattern classification based on textures.

\section{PROPOSED SCHEME}

Texture analysis is an important and useful area of study in machine vision. Automated inspections, medical image processing, document processing, remote sensing etc. are the areas where texture can play important role. Most natural surfaces exhibit texture and successful vision system must be able to deal with textured world surrounding it



Figure 2. Block diagram for texture pattern classification

The working of proposed scheme is described in the following sections. 


\subsection{Preparation of data base}

In this paper, 10 textures from Brodatz database of size $640 \times 640$ is selected. The database is prepared by cropping five numbers of 256X256 images from each texture. The mean feature vector (MFV) is derived from this crop, which indicates one class. Thus we have ten classes and the problem is to find to which class the query image belongs.

\subsection{Feature Vector derivation}

Two feature vectors are derived namely, query feature vector (QFV) and mean feature vector (MFV).

QFV: A query image is one of the crops of size 256x256 from these 10 textures (which is not the part of the data base). The query image is decomposed up to 3rd level, using DWT [5]. Two features, standard deviation and entropy are calculated, of 12 parts obtained after $3^{\text {rd }}$ level decomposition. Thus the QFV is formed

$$
\mathrm{QFV}=[\mathrm{Sd} \text { Ep1 Ep2 Ep3 ...........Ep12] }
$$

MFV: The mean feature vector (MFV) is derived in the same way above but it is the mean value of the 5 different crops from the same texture. Thus we have ten MFVs each representing one class.

$$
\mathrm{MFV}=[\mathrm{Sd} \text { Ep1 Ep2 Ep3 ….......Ep12] }
$$

\subsection{Classification}

For classification distance similarity is used. The different distances used in this scheme are:
1. Euclidean distance
2. Canberra distance
3. Manhattan distance
4. Bray Curtis distance.

The efficiency of classification is calculated for each distance and is compared. The time required for each retrieval using every distance method is also calculated. The efficiency is calculated as

$$
\eta(\%)=\frac{\text { Total no. of samples }- \text { No. of misclassified samples }}{\text { Total no. of samples }} \times 100
$$

\section{RESULTS}

In this paper we have used 10 textures (D1, D2, D88, D67, D27, D77, D90, D8, D9, D3) obtained from Brodatz texture album [10]as shown in fig 3.1. The number below is representing the class number.

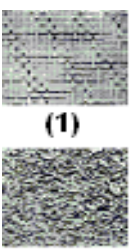

(5)

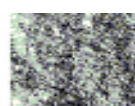

(2)

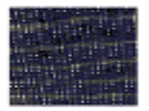

(6)

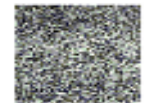

(9)

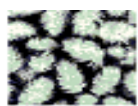

(3)

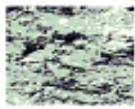

(7)

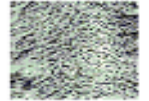

(10)
Figure 3.1 Database Images representing 10 classes

Non-overlapping crops of $256 \times 256$ sizes constructed a database of 50 images from 10 texture classes. For decomposition we have used Daubechies wavelet ' $\mathrm{db} 1$ '. The features used for the classification are standard deviation \& entropy. For different distance feature measures the classification efficiency and the retrieval time is calculated. Table 1 represents the result obtained for 50 query samples.

Table 1

\begin{tabular}{|c|c|c|c|c|}
\hline $\begin{array}{c}\text { Distance } \\
\text { Used }\end{array}$ & $\begin{array}{c}\text { Eucl- } \\
\text { idean }\end{array}$ & $\begin{array}{c}\text { Manh- } \\
\text { attan }\end{array}$ & $\begin{array}{c}\text { Canb- } \\
\text { erra }\end{array}$ & $\begin{array}{c}\text { Bray } \\
\text { Curtis }\end{array}$ \\
\hline Efficiency & $64 \%$ & $70 \%$ & $96 \%$ & $96 \%$ \\
\hline $\begin{array}{c}\text { Av. Time } \\
\text { For Retrieval }\end{array}$ & 0.36 & $\begin{array}{c}0.34 \\
\mathrm{sec}\end{array}$ & $\begin{array}{c}0.42 \\
\mathrm{sec}\end{array}$ & $\begin{array}{c}0.48 \\
\mathrm{sec}\end{array}$ \\
\hline
\end{tabular}

Some experimental results of pattern classification are shown below in figure 3.2 for different distances used.

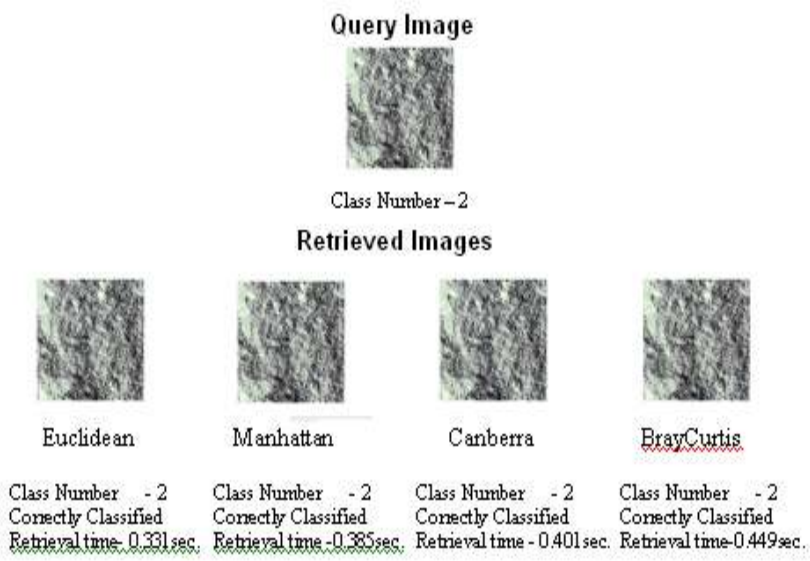




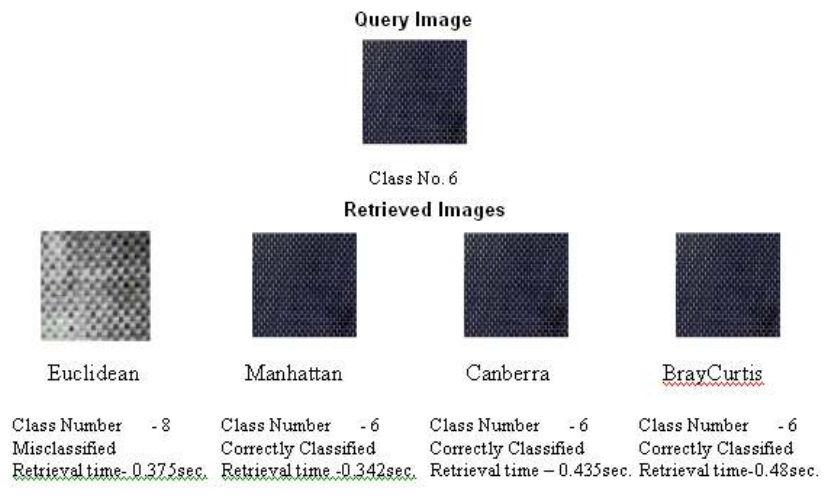

Figure 3.2 Experimental Results

\section{Conclusion}

In this paper we introduced some fundamental techniques for texture image retrieval, including visual content description, similarity distance measures and system performance evaluation. Our emphasis is on visual feature description techniques. General visual features most widely used in image retrieval are entropy and standard deviation. There are various ways to calculate the similarity distances between visual features.

Some basic distances like Euclidean, Manhattan, Canberra \& Bray Curtis are used here for image retrieval. Matching is done using these distances and the efficiency is calculated. The Canberra and Bray Curtis distance gives satisfactory results as compared to other distances, because these distances are calculated using normalization method. But the retrieval time is increased in these distances.

In this paper we have used simple distance methods. We can make use of neural networks or fuzzy logic for pattern classification. The work carried out here is based on the same size of images. So we can work for rotation invariant and scale invariant texture classification. Also the textures used here are only gray scale images. There is lot of scope for colored texture classification using various other features.

\section{REFERENCES}

[1] R. M. Haralick, "Statistical and Structural Approaches to Texture", Proceeding of IEEE vol.67, no. 5, pp.786-804, 1979.

[2] R. M. Haralick, K. Shanmugam and I. Dienstein, "Textural features for image classification", IEEE Transaction on systems, Man and Cybernatics, vol. 3, pp. 610-621, 1973.

[3] R. W. EHRICH AND J. P. Foith, "A View of Texture Topology and Texture Descriptors", Computer Graphics and Image Processing, Vol. 8, pp. 174-202, 1978.

[4] J. Portilla and E. P. Simoncelli, "A Parametric Texture Model based on Joint Statistics of Complex Wavelet Coefficients", Int'l Journal of Computer Vision. 40 (1): 4971, October, 2000

[5] Hawkins, J. K., "Textural Properties for Pattern Recognition", In Picture Processing and Psychopictoris, B. Lipkin and A. Rosenfeld (editors), Academic Press, NewYork, 1969.

[6] The Daubechies Wavelet Transform, Kristian Sandberg Dept. of Applied Mathematics, University of Colorado at Boulder, LaTeX2HTML translator Version 98.1 release (February 19th, 1998).

[7] Raghuvieer M. Rao, Ajit S. Bopardikar, "Wavelet Transforms", Pearson Education Asia, pp 1-5, 183-185.

[8] Gonzalez, R. C., R. E. Woods, S. L. Eddins, "Digital Image Processing Using MATLAB", New Jersey, Prentice Hall, 2003, Chapter 11.

[9] Manjunath B. S. and W. Y. Ma, "Texture Features For Browsing and Retrieval of Image Data", IEEE Transactions on Pattern Analysis and Machine Intelligence, vol. 18, no. 8, p. 837 - 842, Aug 1996.

[10] http://www.ux.uis.no/ tranden/Brodatz.html 